\title{
Stigma, Discrimination, and Social Exclusion of the Mentally Ill: the Case of Ghana
}

\author{
Magnus Mfoafo-M'Carthy ${ }^{1} \cdot$ Marie-Antoinette Sossou $^{2}$ (D)
}

Published online: 27 October 2017

(C) Springer International Publishing AG 2017

\begin{abstract}
The authors examine treatment and society's perception of mental illness through the lenses of stigma, discrimination and social exclusion, and human rights. We argue that being diagnosed with mental illness in a developing country has a significant impact on virtually every area of one's life. The challenges faced by individuals with mental illness in Ghana are due to the prevailing culture and societal practices that tend to stigmatize, discriminate, and socially exclude these individuals from living normal lives. The lack of resources and political will continue to compound this problem. The paper argues for the importance of embracing individual, cultural and institutional training and change in attitudes, perceptions, and funding of services to address these issues of mental illness.
\end{abstract}

Keywords Mental illness - Stigma - Discrimination · Ghana · Human rights

Mental health is defined as a state of well-being in which every individual realizes his or her own potential, can cope with the normal stresses of life, can work productively and fruitfully, and is able to make a contribution to her or his community (WHO 2014). Mental illness or mental disorders

Marie-Antoinette Sossou

msoss2@uky.edu

Magnus Mfoafo-M'Carthy

mmfoafomcarthy@wlu.ca

1 Lyle S. Hallman Faculty of Social Work, Wilfrid Laurier University, 120 Duke Street West, Kitchener, ON N2H 3W8, Canada

2 College of Social Work, University of Kentucky, Lexington, KY, USA are generally characterized by a combination of abnormal thoughts, perceptions, emotions, behavior, and relationships with others (WHO 2017). Mental illness is a major health condition that affects individuals, families, and communities in both the developed and developing world. Statistics from the World Health Organization (2013) indicate that one quarter of the world's population will experience mental illness in their lifetime. Mental illness affects people of different backgrounds and culture, irrespective of age, gender, race, education, religion, or socio-economic status (Regehr and Glancy 2014). According to research, individuals diagnosed with mental illness find themselves stigmatized by family, society, and the community at large. A large majority of individuals with the illness find themselves discriminated against due to society's perception of mental illness (Regehr and Glancy 2014).

In Ghana and most African countries, the belief and practice of witchcraft and its relation to mental illnesses and seeking of mental health treatments from native doctors/traditional healings and spiritual/prayer camps healers are common and these traditional/native healers are regarded as part of the wider cultural belief systems (Amo-Asare 1994; Harding 1975). These cultural beliefs and myths surrounding mental illness are seen as curses from the gods for evil deeds of individuals or from family misdeeds. Twumasi (1979) identified two medical systems in Ghana, namely the western and traditional systems. According to him, there were different groups of traditional healers; the herbalists who mostly used herbs for healing and the cult healers who deal with witchcraft and disasters in social living pertaining to their followers and the leaders of syncretic or spiritual churches who used prayers and other religious ritualization in healing members of their faith. Twumasi (1979) asserts that the common essential element in traditional medicine is the utilization of magicoreligious acts and symbols in diagnosis and therapeutics. 
In this paper, the authors attempt to explore challenges faced by individuals diagnosed with mental illness in Ghana. Due to illiteracy, cultural beliefs, poverty and limited modern specialized mental services, the mentally ill are stigmatized, discriminated against, and socially excluded from families, communities, and the society. It is difficult for these individuals to contribute meaningfully to the society. The authors describe the experiences of an individual diagnosed with mental illness and the challenges she faced pertaining to her illness to illustrate and showcase the fate of most persons with mental illness including their experiences of stigma, discrimination and abuse of human rights in Ghana. We hope this paper will contribute to the dearth of literature focusing on the challenges faced by people with mental disabilities in Ghana and other African societies.

\section{Mental Health in Ghana}

The WHO (2013) reports that low and middle income countries in Africa (including Ghana), Asia, and Latin America bear a disproportionate burden of mental disorders. The report estimates that $10 \%$ of Ghanaians live with mild mental illness while 1-3\% lives with severe mental illnesses such as schizophrenia (WHO 2013). According to Sottie et al. (2016), Ghana has not developed the infrastructure and public services, including mental health care, to keep pace with population growth. Ghana has only three governmental or public psychiatric hospitals available for a population of about 27 million people. These facilities are the Pantang Psychiatric Hospital (in Accra), Accra Psychiatric Hospital (in Accra), and Ankaful Psychiatric Hospital (in Cape Coast), all of which are underfunded, overcrowded, and located in the urban and southern part of the country (Ewusi-Mensah 2001; Fournier 2011). In addition to these, there are three private psychiatric hospitals, one in Tema and two in Kumasi in the Ashanti Region (Basic Needs n.d.).

Similar to general healthcare and other public services, psychiatric services tend to be concentrated within the urban centers. In this regard, the majority of the poor who live in the rural areas and far from the major cities face the greatest challenges in accessing mental health care (Read et al. 2009). Health posts run by Community Psychiatric nurses (CPNs) have been instrumental in providing primary psychiatric services across the country and in referring serious cases to the hospitals available (Ewusi-Mensah 2001). There is one qualified psychiatrist per one million people in Ghana (AppiahPoku et al. 2004). This situation forces patients suffering from various forms of mental disorders to seek help from traditional healers, pastors, and Muslim Mallams in their communities (Appiah-Poku et al. 2004).

Read et al. (2009) suggest that traditional healers and pastors of the Pentecostal churches continue to deal with the greatest proportion of persons with mental disorders in Ghana. Ofori-Atta et al. (2010) conclude that in countries such as Ghana, many persons in need of mental health treatment do not reach psychiatric services at all, but seek care from informal community mental health services such as traditional and faith healers and family members. As of 2011, clinicians working in mental health in Ghana comprised of 18 psychiatrists, 1256 nurses, 19 psychologists, 21 social workers, four occupational therapists, and 546 other mental health workers (Roberts et al. 2014).

These include auxiliary staff, health assistants, medical assistants, and paraprofessional psychosocial counselors. Statistically, it is believed that there are 4.43 mental health nurses per 100,000 residents. A previous 2008 study revealed that Ghana had ten psychiatrists compared to 13,074 physicians (Doku et al. 2012). The annual budget allotted to mental health is $1.4 \%$ of the health budget (Roberts et al. 2014) compared to $10 \%$ or more of national health budgets allotted to mental health in the Western world (Lurie 2014).

There is no accurate epidemiological data on persons with mental health disorders in Ghana since the traditional and faith healers do not normally keep records on their patients (Sottie et al. 2016). Consequently, the limited data discussed in national documents would not include the contributions of family members, religious healers, and traditional healers, who form the backbone of care for the mentally ill in many countries of sub-Saharan Africa, including Ghana (Read et al. 2009). Since the majority of persons with mental disorders use mental health services outside formal health institutions, concerns have been raised regarding abuses that mentally ill persons experience during their treatment and residence at these informal settings (Appiah-Poku et al. 2004).

\section{Abuse of Human Rights and Effects of Social Exclusion, Stigma, and Discrimination on Persons with Mental Illness}

According to the Universal Declaration of Human Rights (UDHR), every person has the right to freedom, justice, and peace (United Nations 1948). Article 25 (section 1) states that "everyone has the right to a standard of living adequate for the health and well-being of himself and of his family, including food, clothing, housing and medical care and necessary social services, and the right to security in the event of unemployment, sickness, disability, widowhood, old age or other lack of livelihood in circumstances beyond his or her control."

Gostin and Gable (2009) affirmed that human rights of persons with mental disabilities are impacted by three important relationships between mental health and human rights. First, coercive mental health policies infringe on human rights; second, invasions of human rights harm mental health; 
and third, positive promotion of mental health and human rights has mutually reinforcing and synergistic results.

People with mental health disorders in Ghana do not have access to basic mental health care and treatment. Furthermore, the absence of effective community-based mental health services leaves patients to be locked up in psychiatric institutions and other places with limited facilities where they are subjected to traditional treatments that are deemed inhumane. Unfortunately, patients are exposed to gross human rights abuses and violations in these institutions and healing and prayer camps. These human rights violations include: denial of employment, marriage, having children, and education; malnutrition, physical abuse and personal negligence, and stigma and discrimination. The World Health Organization (2011) refers to the abusive conditions endured by people with mental health conditions as a hidden human rights emergency.

Social exclusion is a situation experienced by individuals primarily due to marginalization from society through economic deprivation, social isolation and fragmentation of social relations (Figueiredo and de Haan 1998). Atkinson (1998) and Kleinman (1998) believed that exclusion refers to an act by an agent or agents, such as individuals, groups, institutions or markets that have the power to exclude people from various spheres of life.

People with mental illness in Ghana suffer from various forms of deprivation and social exclusion such as lack of income, which is usually related to unemployment; lack of proper housing; poor physical health and under-nourishment; psychological problems; and unpleasant and insecure family and personal relationships. According to Goffman (1963), stigma is deeply discrediting and causes the person experiencing it to be perceived as different and as less pleasant than others. Link and Phelan (2001) assert that the unpleasant phenomenon of stigma is accompanied by stereotyping, rejection, loss of status, and discrimination.

The theoretical perspective undergirding the issues of stigma and discrimination is derived from the social model of disability concept. This model posits that any form of disability, including mental illness, is a social construction created by society to impose hindrances to the full participation of persons with different abilities (Hughes and Patterson 1997; Harris 2000; Swain and French 2000). Such hindrances include negative attitudes, physical impediments, and institutional, communication, and social barriers. According to the social model, disability is differentiated from impairment by exclusionary societal practices that isolate and stigmatize individuals with a disability (Goodley 1997; Hughes and Patterson 1997). This model adopts a civil rights perspective on the social and civic participation of persons with a disability (Marks 1997a, b; Munn 1997).

According to Hergenrather et al. (2005), stigmatization can greatly impact people's quality of life and utilization of necessary community resources. Furthermore, particular factors such as gender, age, culture, and experience of those working with individuals with mental disabilities can greatly influence an individual's perception of persons with such disabilities (Tervo et al. 2002).

Ciftci et al. (2013) defined discrimination as the behavioral component of stigma which occurs when people act on the basis of prejudiced attitudes or beliefs. Goreczny et al. (2011) describe discrimination as the way persons are treated, intentionally or unintentionally, due to stigma. According to Baffoe (2013) and Dako-Gyeke and Asumang (2013), discrimination may lead to societal exclusion, bullying, aggression, ridicule, and devaluation of the self-worth of people.

Dako-Gyeke and Asumang (2013) assert that discrimination and stigma are devastating experiences for persons with mental health disabilities in Ghana because in most communities, people tend to have strong views about mental illness, which are often based on stereotypes and traditional beliefs. Dako-Gyeke and Asumang (2013) conducted a qualitative study on stigmatization and discrimination among people with mental illnesses in Ghana. The authors found that there are high levels of negative perceptions by family members of mental disorders in general which resulted in much stigmatization and discrimination against persons with mental illness. Secondly, they discovered that persons with mental disorders suffer from isolation, stigmatization, and discrimination due to misconceptions about mental illness. They are robbed of opportunities for quality of life and purposeful interaction in their communities, with family members and friends.

Their study found that persons with mental disorders also experienced stigmatization, and discrimination from their employers, colleagues and coworkers due to negative perceptions and cultural beliefs and misunderstanding about the etiology of mental disorders in general (Dako-Gyeke and Asumang 2013). Arguably, these are the daily lived experiences of most persons with mental illness in Ghana. There have been reports of individuals whose families and loved ones abandoned them at mental institutions due to the stigma associated with the illness (Barke et al. 2011). Using the experience of Ms. Appiah Danquah, an individual diagnosed with mental illness in Ghana, and the challenges she and her family faced during the course of her diagnosis and treatment as she continued to be stigmatized, discriminated against, and socially excluded. The plight of Ms. Danquah as featured in a documentary produced by a private film maker, Creative Storm, is not unique as most individuals with mental illness in Ghana are ostracized and treated with disdain. This documentary shed light on some of the issues of other mental health patients and individuals in Ghana and also supports the literature discussed in this paper.

\section{Case Example}

Ms. Doris Appiah Danquah attended one of the premier high schools in Ghana, Achimota School in Accra. Due to her 
excellent academic performance, she was eventually admitted to the Medical School at the University of Ghana. However, she had to eventually drop out of Ghana Medical School due to bouts of depression which made it difficult for her to focus on her studies. For a period of approximately 22 years, Ms. Appiah Danquah was moved from one place to another while being drugged, stigmatized, chained, and beaten at spiritual camps and at fetish shrines. At one point, she was made to understand that her mother had cursed her and caused her to be mentally ill.

Also, she was told that her condition was a result of not serving God well, and so she would never recover. In one incident, a group of children who knew her bought her food, but then threw the plate away after she had finished eating due to their belief that her illness was contagious. Ms. Appiah Danquah eventually enrolled in a nursing program because she could not go back to study medicine. Upon graduation, the difficulty she experienced in attempting to find work in her field could be attributed to stigma and discrimination. Ms. Appiah Danquah is currently an influential advocate for the rights of the mentally ill in Ghana (DFID 2014).

Numerous factors contribute to this behavior, including the lack of psychosocial approach to the treatment of mental illness, poverty, ignorance, illiteracy, and the continuous prevalence of entrenched cultural myths and practices toward mental illness. The Ghanaian society's perception of mental illness as a curse and a form of weakness warrants the shunning and maltreatment of the mentally ill population as less deserving and socially excluded from their normal involvement in activities such as employment or, having a family.

\section{Suggestions and Way Forward}

A new Mental Health Law was passed in Ghana in March 2012 after several years of advocacy and lobbying. The law reflects international human rights and standards for best practices and at the same time takes into consideration local conditions and requirements (Sottie et al. 2016). It places emphasis on community-based treatment and discourages institutionalization. The law also acknowledges the work of informal mental health care service providers such as traditional and faith-based healers and regulates their practices to avoid human rights abuses (Sottie et al. 2016). The new Mental Health Law (Act 846 of 2012) contains specific provisions that relates to the rights of persons with mental disorders, the protection of vulnerable groups and a visiting committee to monitor human rights conditions in mental health facilities. There are also provisions for the setting up of a tribunal that has the mandate to investigate complaints and review involuntary admission to mental health facilities and treatment (Sottie et al. 2016).
The law would also fight discrimination and stigmatization of people with mental disorders and makes possible for patients to access mental health services within their communities. Hence, the emphasis of this law is on the implementation of community-based mental health treatment programs (Government of Ghana, 2012). Additionally, the law is to provide education, For example, this law is modeled on examples of Assertive Community Treatment Teams (ACTT) and Community Treatment Orders (CTOs) in Canada and the USA. The purpose is to work closely with clients and their families in the communities (Mfoafo-M'Carthy and Sottie 2015). The government has also proposed working in collaboration with faith-based healers and traditional medicine men and fetish priests as a way forward in providing social education and understanding of the etiology of mental disorders in the country.

\section{Recommendations}

Due to challenges faced by individuals diagnosed with mental illness, it is imperative that steps are taken to explore and resolve issues of social exclusion and marginalization. A crucial aspect of the way forward is exploring cultural and social norms in Ghana with regard to mental illness. Individual perceptions of mental illness should be addressed through informal and formal social education and training of the public and traditional healers. Alongside this initiative, community leaders including chiefs and queen mothers (female community leader nominated to serve alongside the chief or the female version of a chief) could be encouraged to provide this education by conveying information about mental health to their communities.

This system of educating community leaders has already been utilized in Ghana and other developing African countries regarding child welfare, and with some positive outcomes. Also, the creation of users' or consumer networks for individuals with mental health disorders could also be a positive way of empowering individuals with mental illness. This network has been utilized in African countries like Zambia, Uganda, Tanzania, Kenya, and South Africa with tremendous success (Katontoka 2007). The essence of the network is to encourage members to engage in community activities such as awareness campaigns and self-help projects which often result in the development and improvement of mental health services.

It should be borne in mind that being diagnosed with mental illness does not have to shorten one's future or ability to contribute to the economy. The government of Ghana in conjunction with non-governmental organizations (NGOs) could set up training programs for those hospitalized so that upon discharge they could be gainfully employed or engage in selfemployment with some financial assistance. In addition, religious groups and NGOs could play a significant role in the re- 
integration of these individuals into society by assisting with the process of adjustment (Ame and Mfoafo-M'Carthy 2016).

There is the need for proper training and recruitment of psychiatric and other mental health personnel, such as doctors, nurses, and social workers and in addition to increased funding for mental health services to augment the challenges of inhuman treatment, stigma, and discrimination associated with mental illness in the country (Roberts et al. 2014).

It is therefore incumbent on the government to create awareness on and to provide individuals with mental illness the opportunity to contribute to the economic growth of the country. The involvement and inclusion of trained mental health social workers in the campaign against stigma, discrimination and social exclusion, and engaging in social education of individuals, families, groups, and communities on issues of mental health and mental illness should be given priority in both the training and recruitment of social workers in the country.

It is evident that social workers are trained professionals interested in improving and enhancing the quality of life of people, including the very vulnerable populations, hence the importance of their roles and responsibilities toward clients and the society should be included in any effective mental health improvement in Ghana. Social work professionals and other helping professionals such as psychiatrists, nurses, and community nurses among others are crucial to delivering and maintaining excellent mental health services in Ghana. The practice of quality social work can transform the lives of people with mental health conditions and should be an essential part of any effective multidisciplinary and multiagency treatment programs in the country.

\section{Conclusion}

According to the Mental Health and Development report (WHO 2010), mental health is a development issue and people with mental disorders constitute a vulnerable group who need to be targeted in development assistance. The high unmet need for mental health services is remarkably similar in most African countries. Mental health expenditures in these countries are very low compared to the amount of money spent on defense and national security in most of these countries. The traditional mental health care institutions in these countries lack the money to expand, buy needed medications, and find and train sufficient doctors, nurses' social workers, and other caregivers.

Additionally, individuals with mental illness continue to face stigma, social exclusion, discrimination, and violation of their human rights in the country. This is mainly due to the myths and cultural beliefs associated with mental illness. Furthermore, stigma and discrimination may have contributed negatively to the economy as individuals with mental illness have been unable to contribute their quota to the national development of the country. Unfortunately, Ms. Appiah Danquah's experience bears witness to the challenges faced by other patients in the Ghanaian society. It is therefore important for steps to be taken to address these issues of national development, stigma, discrimination and social exclusion from individual, and institutional levels in Ghana. It is obvious that psychiatry as a discipline is underfunded by the government in comparison to other professional disciplines. Hence, institutional training and change of attitudes, perceptions, and direct engagement with various non-governmental organizations would be a step in the right direction.

Considering the fact that poverty and lack of education are attributed to these problems in Ghana (Ame and MfoafoM'Carthy 2016), funding is needed to train more mental health professionals including psychiatrists, psychologists, nurses, social workers, and occupational therapists to work with this population and also provide social education about mental health in the communities. Finally, there should be a strong political will to ensure the establishment of programs which could contribute toward addressing these problems and issues surrounding mental health and stigma in Ghana.

\section{References}

Ame, R., \& Mfoafo-M'Carthy, M. (2016). Mental health law in Ghana: the rights of children with mental disorders. Social Development Issues, 38(1), 1-14.

Amo-Asare, A. (1994). A study of mental health care in Ghana: University of Ghana, Legon: A dissertation submitted to the Department of Community Health.

Appiah-Poku, J., Laugharne, R., Mensah, E., Osei, Y., \& Burns, T. (2004). Previous help sought by patients presenting to mental health services in Kumasi, Ghana. Social Psychiatry and Psychiatric Epidemiology, 39(3), 208-211.

Atkinson, A. B. (1998). Social exclusion, poverty and unemployment. In A. B. Atkinson \& J. Hills (eds.), Exclusion, employment and opportunity. Case Paper no. 4. London: Centre for Analysis of Social Exclusion, London School of Economics.

Baffoe, M. (2013). Stigma, discrimination \& marginalization: gateways to oppression of persons with disabilities in Ghana, West Africa. Journal of Educational and Social Research, 3(1), 187-198.

Barke, A., Nyarko, S., \& Klecha, D. (2011). The stigma of mental illness in southern Ghana: attitudes of the urban population and patients' views. Social Psychiatry Epidemiology, 46, 1191-1202. https://doi. org/10.1007/s00127-010-0290-3.

Basic Needs (n.d.). Ghana the passage of the mental health bill into law. Retrieved from http://www.basicneeds.org/html/newsstory.asp? newsstory $=155$.

Ciftci, A., Jones, N., \& Corrigan, P. W. (2013). Mental health stigma in the Muslim community. Journal of Muslim Mental Health, 7(1), 1732.

Dako-Gyeke, M., \& Asumang, S. (2013). Stigmatization and discrimination Experiences of persons with mental illness: insights from a qualitative study in Southern Ghana. Social Work \& Society, International Online Journal, 11(1) Retrieved from http://www. socwork.net/sws/article/view/362/712. 
DFID (2014). Still I arise: The story of Doris Appiah Danquah. Retrieved from https://vimeo.com/109473357.

Doku, V., Wusu-Takyi, A., \& Awakame, J. (2012). Implementing the mental health act in Ghana: Any challenges ahead? Ghana Medical Journal, 46(4), 241-250.

Ewusi-Mensah, I. (2001). Post-colonial psychiatric care in Ghana. Psychiatrist Bulletin, 25, 228-229. https://doi.org/10.1192/pb.25.6. 228.

Figueiredo, J. B., \& de Haan, A. (1998). Social exclusion: an ILO perspective. Geneva: International Institute for Labor Studies.

Fournier, O. A. (2011). The status of mental health care in Ghana, WestAfrica and signs of progress in the greater Accra region. Berkeley Undergraduate Journal, 24(3), 9-34.

Goffman, E. (1963). Stigma: Notes on the management of spoiled identity. New York: Simon \& Schuster, Inc..

Goodley, D. (1997). Locating self-advocacy in models of disability: understanding disability in the support of self-social workers with learning disabilities. Disability and Society, 12, 367-379.

Goreczny, A. J., Bender, E. E., Caruso, G., \& Feinstein, C. S. (2011). Attitudes toward individuals with disabilities: Results of a recent survey and implications of those results. Research in Developmental Disabilities, 32, 1596-1609.

Gostin, L. O. \& Gable, L. (2009). The human rights of persons with mental disabilities: A global perspective on the application of human rights principles to mental health. Maryland Law Review, 63 (20), 2004 and Wayne State University Law School Research Paper No. 08-31. Retrieved from http://ssrn.com/abstract $=1435443$.

Government of Ghana. (2012). Mental health law. Act 846 of 2012. Accra: Assembly Press Ltd..

Harding, T. W. (1975). Traditional healing methods for mental disorders. WHO Chronicle, 31, 436-440.

Harris, J. (2000). Is there a coherent social conception of disability? Journal of Medical Ethics, 26, 95-100.

Hergenrather, K., Rhodes, S., \& McDaniel, R. (2005). Correlates of job placement: Public rehabilitation counselors and consumers living with AIDS. Rehabilitation Counseling Bulletin, 48(3), 94-115.

Hughes, B., \& Patterson, K. (1997). The social model of disability and the disappearing body: Towards sociology of impairment. Disability \& Society, 12(3), 325-340.

Katontoka, S. (2007). Users' networks for Africans with mental disorders. The Lancet, 370(9591), 919-920.

Kleinman, M. (1998). Include me out? The new politics of place and poverty. Case paper No. 11. London: Centre for Analysis of Social Exclusion, London School of Economics. Retrieved from http:// sticerd.lse.ac.uk/dps/case/cp/paper11.pdf.

Link, B. G., \& Phelan, J. (2001). Stigma and its public health implications. Paper presented at stigma and global health. Bethesda: An international Conference.

Lurie, S. (2014). Why can't Canada spend more on mental health? Health, 6, 684-690. https://doi.org/10.4236/health.2014.68089.

Marks, D. (1997a). Models of disability. Disability and Rehabilitation, 19, 85-91.

Marks, D. (1997b). Models of disability. Who needs models? Disability and Rehabilitation, 19, 492-495.
Mfoafo-M'Carthy, M., \& Sottie, C. A. (2015). Current practices in community treatment of the mentally ill in Ontario, Canada: learning opportunities for improving Ghana's mental health system. Journal of Culture and Mental Health, 8(4), 359-369.

Munn, P. (1997). Models of disability for children (comment). Disability and Rehabilitation, 19, 484-486.

Ofori-Atta, A., Read, U. M., \& Lund, C. (2010). A situation analysis of mental health services and legislation in Ghana: challenges for transformation. African Journal of Psychiatry, 13(2), 99-108.

Read, U. M., Adiibokah, E., \& Nyame, S. (2009). Local suffering and the global discourse of mental health and human rights: an ethnographic study of responses to mental illness in rural Ghana. Globalization and Health, 5(13), 1-16. https://doi.org/10.1186/1744-8603-5-13.

Regehr, C., \& Glancy, G. (2014). Mental health social work practice in Canada (2nd. Ed.). Toronto: Oxford University Press.

Roberts, M., Mogan, C., \& Asare, J. B. (2014). An overview of Ghana's mental health system: Results from an assessment using world health organization's assessment instrument for mental health systems (WHO-AIMS). International Journal of Mental Health Systems, 8, 16. https://doi.org/10.1186/1752-4458-8-16.

Sottie, C., Dako-Gyeke, M., Asampong, E., \& Ackumey, M. (2016). Mental health care and service in Ghana. In M. A. Sossou \& T. Modie-Moroka (Eds.), Mental health conditions and services in selected African countries: Implications for social work and human services professions (pp. 55-76). New York: Nova Science Publishers, Inc.

Swain, J., \& French, S. (2000). Towards an affirmation model of disability. Disability \& Society, 15(4), 569-582.

Tervo, R., Azuma, S., Palmer, G., \& Redinius, P. (2002). Medical students' attitudes toward persons with disability: a comparative study. Archives of Physical Medicine and Rehabilitation, 83, 1537-1542.

Twumasi, P. A. (1979). A social history of the Ghanaian pluralistic medical system. Social Science and Medicine, 13(B), 349-356.

United Nations (1948). Universal declaration of human rights. Retrieved from http://www.ohchr.org/EN/UDHR/Documents/UDHR Translations/eng.pdf.

World Health Organization. (2010). Mental health development report: Targeting people with mental health conditions as a vulnerable group. Geneva: World Health Organization and Mental Health and Poverty Project.

World Health Organization, (2011). Mental health and development. Retrieved from http://www.who.int/topics/mental health/en.

World Health Organization. (2013). Comprehensive mental health action plan 2013-2020. Geneva: World Health Organization Retrieved from http://apps.who.int/gb/ebwha/pdf_files/WHA66/A66 R8-en. pdf?ua $=1$.

World Health Organization (2014). Mental health: A state of well-being. Retrieved from http://www.who.int/features/factfiles/mental health/ en/.

World Health Organization (2017). Mental disorders: Fact sheet. Retrieved from http://www.who.int/mediacentre/factsheets/fs396/ en/. 